\title{
Twinning hierarchy, shape memory, and superelasticity demonstrated by molecular dynamics
}

\author{
M. Zelazny, R. Richardson, and G. J. Ackland \\ School of Physics, SUPA and CSEC, The University of Edinburgh, Mayfield Road, Edinburgh, EH9 3JZ, United Kingdom
}

(Received 10 June 2011; published 18 October 2011)

\begin{abstract}
A martensitic phase transition exhibiting shape memory, transformation-induced plasticity, or superelasticity typically involves a transformation between a high temperature, high symmetry phase and a low temperature, low symmetry phase. There have been numerous attempts using molecular dynamics to simulate the shape memory behavior, where the memory is stored in a twinned martensite and deformation occurs by motion of twin boundaries. However, the 3D case has always proved elusive, because suitable interatomic potentials to produce a unique low temperature phase are difficult to obtain. Here we present a study in which the binary Morse potential is tuned specifically to maximize the difference between $\mathrm{L}_{0}$ and $\mathrm{B} 19$ (Strukturbericht notation, spacegroups $P 4 / \mathrm{mmm}$ and $\mathrm{Pmma}$ ) structures. The twinned structure of martensite has been induced by gradually cooling the sample below the transition temperature. A bar-shaped sample was plastically deformed in the martensite phase, and on reheating above the transition temperature the initial shape was recovered. The effect of the shear-induced phase transition on the nanostructure of resulting martensite has also been investigated. An unusual discovery is that of a hierarchy of twins: nanotwins accommodate the mismatch between austenite and martensite at the habit plane, while dynamically created macrotwins are responsible for the deformation behavior and shape memory.
\end{abstract}

DOI: 10.1103/PhysRevB.84.144113

PACS number(s): $62.20 . f g, 62.20 . f q, 64.70 . K-$

\section{INTRODUCTION}

Shape memory alloys (SMAs) are a fascinating class of materials, finding applications ranging from medicine to aerospace, so there is no shortage of motivation toward better understanding their behavior and the structures involved. ${ }^{1,2}$ The interest in SMAs stems from the two characteristic properties that define them. The first of these is that the alloy can sustain large deformations at low temperatures, and recover this deformation completely on heating above a certain transition temperature (the SMA remembers its high temperature state). The second property is superelasticity, in which the material can completely recover from large strains once the stress being applied is removed.

Standard theory has it that underlying both the shape memory and superelastic effects is the martensitic transformation (MT). This is a displacive (diffusionless), first-order phase transition from a high temperature, high symmetry phase conventionally called austenite to a lower temperature, lower symmetry phase called martensite. ${ }^{1,3,4}$ The most important feature of SMAs is that, while the structure and dimensions can change appreciably during the MT, the relative positions of the atoms remain the same. Put simply, the atoms remember who their neighbors are. The key to this memory lies in the groupsubgroup relation: the martensite must have a crystallographic symmetry which is a subgroup of the austenite structure. The symmetry may break in more than one equivalent direction, so multiple orientations, variants, of martensite can correspond to a single orientation of austenite. These variants are separated by twin boundaries across which the relationship of local order to a unique austenite is maintained. The Eriksen-Pitteri neighborhood (EPN) of the austenite refers to the set of martensitic crystal structures with this correspondence. This is clearly useful for shape memory, since the atoms must always return to the same position on reheating of a crystal from martensite to austenite. Diffusion (atoms leaving the EPN) would therefore destroy all memory of the original, high temperature state. In order for the martensite to grow compatibly from the austenite there must be a habit plane common to both phases. Typically this is a high-index or irrational plane determined by the ratios of the lattice parameters. In 3D lattice parameter matching is not possible except in special cases, ${ }^{5}$ and compatibility demands that the martensite phase be twinned. ${ }^{3}$

There are additional requirements for shape memory, notably that deformation should take place by phase transformation or twinning, and not by irreversible mechanisms such as dislocation motion. In SMAs, twinning deformation occurs by a movement of twin boundaries such that each atom remains in the same EPN. In nanomaterials, where dislocation motion is suppressed, we can expect transformation deformation to be dominant. It has been shown that MT in $\mathrm{Zr}$ can act as a source of dislocations, ${ }^{6}$ but in a binary alloy dislocation formation is more difficult: here we will start with dislocation-free samples, and find no evidence of dislocation generation.

Alternate methods of shape memory are also possible: in NiTi the reverse shape memory effect requires memory to be stored in the high symmetry austenite. This must be stored in microstructure of internal stress fields.

Previous molecular dynamics (MD) simulations of shape memory have not found a twinned martensite with well defined group-subgroup relation, ${ }^{7}$ or have had significant surface or finite-size effects ${ }^{8,9}$ which have long been known to affect phase behavior. ${ }^{10}$ One implication of this is that nanowires of materials without phase transitions could still exhibit shape memory driven by surface reorientation. ${ }^{11,12}$

The martensitic transformation can either be temperature driven, as for the shape memory effect, or stress induced. In the latter, a large strain can induce the formation of martensite in high-stress regions, which can accommodate the deformation reversibly and recover (transform back to austenite) once the material is allowed to relax again.

Here we consider atomistic simulations of shape memory and superelasticity, using a MD approach ${ }^{13}$ with a B2 austenite and a B19 martensite, similar to AuCd. 


\section{CRYSTAL STRUCTURES}

B2 is the austenite structure in many of the more common SMAs, such as NiTi, ${ }^{14} \mathrm{NiAl},{ }^{15}$ and AuCd. ${ }^{16}$ This is a biatomic analog of the body centered cubic (bcc) structure. On cooling, the alloy will often transform to martensite via various intermediate or metastable phases, such as the $\omega$ phase in $\mathrm{Zr}$ or the $R$ phase in NiTi. In AuCd the B2-B19 transformation path involves a tetragonal distortion of a doubled unit cell, resulting in martensitic structures such as the orthorhombic $\gamma_{2}^{\prime}$ phase (B19) found in AuCd, or its deformation B19' observed in NiPt, NiPd, and NiTi. ${ }^{17-20}$

B19 can be regarded as a binary analog of hcp, and the B2-B19 transition follows a Burgers-type path similar to bcc-hcp. However, the binary compound does not have hexagonal symmetry, meaning that it is a subgroup of B2 and could exhibit shape memory. This contrasts with elements such as $\mathrm{Zr}$ and $\mathrm{Ti}$, which have been widely modeled in the past. 6,21,22 These elements have the bcc-hcp transition, and hcp is not a subgroup of bcc. Consequently that previous work provides insight into martensitic transitions, but not shape memory effect. Both bcc-hcp and B2-B19 transitions involve a unit cell doubling and atomic shuffle which combine to give 12 variants.

A key aspect which has hindered previous work is the lack of an interatomic potential which avoids metastable low temperature phases. For elements, this means that hcp must be very stable compared with fcc. ${ }^{23}$ For binary compounds it means $\mathrm{B} 19$ should have much lower energy than $\mathrm{L}_{0}$. In most previous studies, parameters have been used to stabilize the $\mathrm{L}_{0}$ phase, and the martensite phase has been described as "close-packed," which on closer inspection ${ }^{24}$ meant a mixture of ABA and ABC stacking (cf. B19 and $\left.\mathrm{L}_{0}\right)^{7-9}$

Here we want to investigate the B2-B19 transformation. In order to do this we need interatomic potentials which gives a significant energy difference between $\mathrm{B} 19$ and $\mathrm{L} 1_{0}$. This will ensure a pure phase martensite, rather than a mixture of $\mathrm{L}_{0}$ and B19 or some kind of random stacking of near-close packed planes.

We are not attempting to simulate a particular material, so we will use a binary Morse potential ${ }^{25}$ :

$$
\begin{gathered}
V(r)=V_{0}(r)-V_{0}\left(r_{\max }\right), \\
V_{0}(r)=\epsilon_{\mathrm{AB}}\left\{1-\exp \left[-\alpha_{\mathrm{AB}}\left(r_{\mathrm{AB}}^{\mathrm{eq}}-r\right)\right]\right\}^{2},
\end{gathered}
$$

where $r$ is the interatomic separation, $\alpha$ is the attraction "range," and $\epsilon$ is depth of the energy well. $r^{\text {eq }}$ is the equilibrium spacing for a dimer, $r_{\max }$ is the range of the potential beyond which $V(r)=0$, and $\mathrm{AB}$ label the species at either end of the bond. In the binary alloy this gives a sevendimensional parameter space and a surprisingly rich phase diagram which has not been fully mapped out. Elliott ${ }^{26-28}$ has investigated the mechanical stability of biatomic crystals against phonon, homogenized continuum and Cauchy-Born (elastic) stability and proposed some parameters which satisfy these stability criteria. By changing the potential, shape memory can be demonstrated using Cauchy-Born kinetics. ${ }^{29}$ Here, however, we are interested in thermodynamic stability, where the entropy emerges from configuration sampling of lattice vibrations at high temperatures.
Although capturing many of the right features when used in preliminary MD and with Cauchy-Born kinetics, Elliott's parametrization gives phase separation and, on MT, a martensitic mixture of fcc-like $\mathrm{L} 1_{0}$ and hcp-like B19. We therefore searched for parametrizations which met the following criteria.

(1) The energies of $\mathrm{B} 2$ and $\mathrm{L}_{0}$ are greater than the energy of B19.

(2) There is a sufficiently large energy difference between the $\mathrm{B} 19$ and $\mathrm{L} 1_{0}$ that $\mathrm{B} 19$ remains the only energetically favorable state at low temperatures.

(3) The alloyed phase, whether B19 and B2, is more energetically favorable than the corresponding single-species phases (single-species hcp and bcc).

To deal with the phase separation problem and to reduce the parameter space we set $\epsilon_{\mathrm{AB}}=\epsilon_{\mathrm{BA}}>\epsilon_{\mathrm{AA}}=\epsilon_{\mathrm{BB}}$. and fixed the values of equilibrium bond length $r_{\mathrm{eq}}$ and potential width $\alpha$ to those used previously. ${ }^{27}$

The parameter $r_{\max }$ has the strongest effect on the phase stability, since it determines which shells of neighbors fall within the potential range. A value between 1.78 and 1.88 is needed to destabilize B2 at $T=0$, while B19 stability requires $r_{\text {max }} \approx 1.8$. We scanned the parameter space to determine which would maximize the $0 \mathrm{~K}$ energy difference between B19 and $\mathrm{L} 1_{0}$ while still having unstable $\mathrm{B} 2$ and phase separation. The fitting process also rejected any potential with a lower free energy for a 50-50 "disordered" bcc structure, represented by the enthalpy of a single disordered 18 000-atom supercell at $0 \mathrm{~K}$ plus the free energy of mixing at $100 \mathrm{~K}$. Other possible phases were not considered in the parametrization process, but the final potential was tested for stability against other phases found in Elliot's work. Based on our recent experience of generating interatomic potentials, ${ }^{30-33}$ we hypothesise that low symmetry phases which are unstable at $0 \mathrm{~K}$ will remain unstable at high $T$, but that high symmetry phases (here, B2) might be entropically stabilized.

From this we were able to determine a range of parameters which meet the requirements above. The exact transformation temperature cannot be fitted in this process, nor the hysteresis, nor can it be guaranteed that the transformation will not be preceded by melting. Each of these essential criteria can only be checked by further testing, and this testing forms the second, more empirical, phase of potential derivation.

By this process we obtained the parameters given in Table I, with which we performed a series of MD simulations heating and cooling the crystal using MOLDY, ${ }^{13}$ which introduces a reduced temperature scale defined in Table I. By monitoring potential energy and analyzing the software ${ }^{34}$ we established that the B19-B2 phase transition lay between $T=70$ and $T=75$ (reduced units). Moreover, at all temperatures $\mathrm{L} 1_{0}$ has higher energy than B19. Using the method of coexistence we determined that the melting temperature was $T=90$, which was assumed to be high enough to exceed the austenitic transition, even in the presence of hysteresis, as described later, this turns out to be correct.

\section{MODELING THE SHAPE MEMORY EFFECT}

In the following we describe simulations of the shape memory effect and visualize the martensitic phase transition. 
TABLE I. Morse potential parameters. The first column indicates which interaction potential was being parametrized (where $\mathrm{Au}$ and $\mathrm{Cd}$ refer to the two atomic species). $r_{\mathrm{eq}}$ and $\alpha$ are taken from Ref. 27 and $\varepsilon$ and $r_{\text {cut }}$ are determined to optimize the SMA properties. Energies and lengths are in scaled units following Ref. 27, but for purposes of calculating temperature and compatibility with MOLDY we use a factor of $2 e / 3 k_{B}=7735.5$. The time step in reduced units is 0.015 and the masses are set to 112 and 197 to replicate $\mathrm{Au}$ and $\mathrm{Cd}$. At $T=0$ these parameters give a B2 lattice parameter of 1.197 and B19 parameters $1.086,1.810$, and 1.732 .

\begin{tabular}{lcccc}
\hline \hline Bond AB & $r_{\mathrm{eq}}$ & $\alpha$ & $\varepsilon$ & $r_{\text {cut }}$ \\
\hline $\mathrm{Au}-\mathrm{Au}$ & 1.0657707400 & 3.7531523900 & 0.0135279 & 1.85 \\
$\mathrm{Cd}-\mathrm{Cd}$ & 1.0924995364 & 6.4073253760 & 0.0135279 & 1.85 \\
$\mathrm{Au}-\mathrm{Cd}$ and Cd-Au & 1.0750728008 & 5.1159326101 & 0.0165279 & 1.85 \\
\hline \hline
\end{tabular}

It is known that boundary conditions can have significant effects on the simulation. Periodic boundary conditions will prevent the habit plane between austenite forming anywhere but perpendicular to the boundary. Surfaces can change the free energy balance between phases. Fixed atoms or surfaces can act as nucleation sites for MT or for cracks.

The martensitic transformation should be free to produce a martensite at any orientation, with a habit plane lying at any angle. Periodic boundary conditions do not allow this freedom, ${ }^{30}$ and simulating sufficient bulk material to represent a polycrystal for the required times is unrealistic. Consequently, we chose a nanorod geometry which it would be experimentally feasible to create. Specifically, we use an 80000 atom $(200 \times 20 \times 5$ B19 cells $)$ bar oriented with the long direction on [110], which is close to the geometrically determined habit plane. The free surfaces of the sample cause two problems: first is that they might shift the phase transformation temperature, in fact this turned out not to be significant. The second was that surface relaxation on setting up the sample can cause significant heating and initiate shock waves. This is a particular problem for B2 samples where one cannot relax at zero temperature. It was resolved by a frequent resetting of the atomic velocities to destroy the wave coherence and maintain temperature to establish a defect-free B2 sample at $T=74$. This was used as the starting configuration for subsequent loading and heating simulations.

\section{A. Reversibility of the transition}

Our first set of investigations concern the mechanism of the MT. The sample was cooled down to $T=29$ by steadily reducing the target temperature in the thermostat ${ }^{35,36}$ at a rate much slower than the equilibration time. A martensitic phase transition started at $T=54$ and was completely finished at $T=53$. A visualization of the phase transition is given in Fig. 1 with atoms identified by local coordination based on the averaged position of the atoms over 500 time steps: this averages over any lattice vibration. ${ }^{34}$ Snapshot 1 was taken at $T=55$, just before the phase transition, the sample is completely austenitic. Fluctuating subcritical nuclei of martensite then begin to appear isotropically, with some suggestion that the nucleation is self-suppressed by its associated strain field, while the strain field also
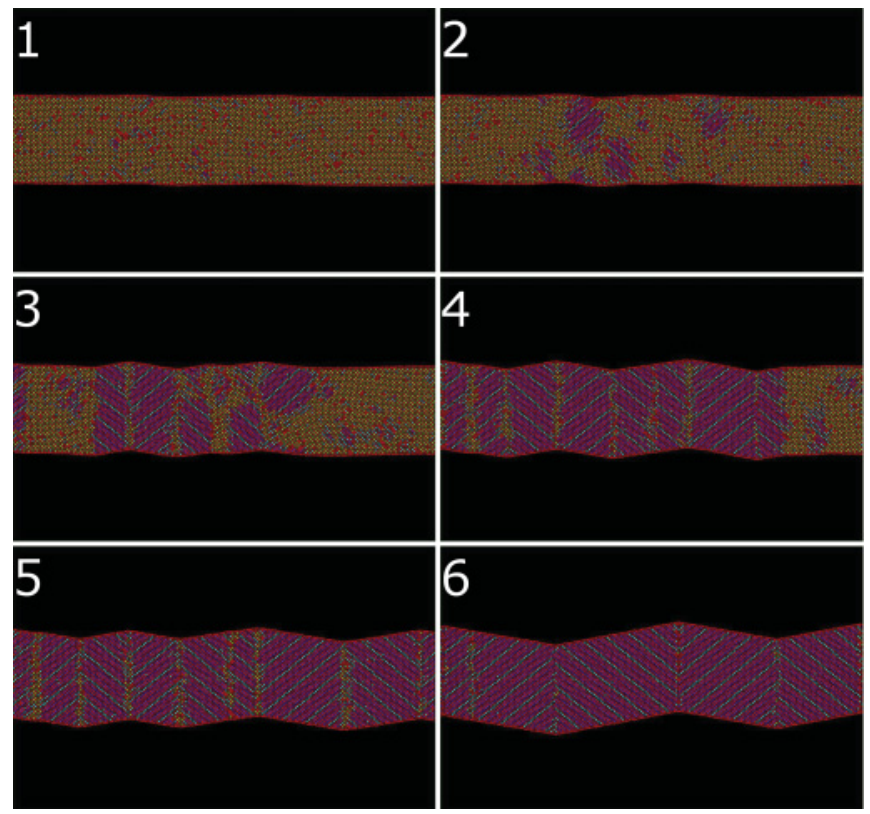

FIG. 1. (Color online) Snapshots of thermally induced B2 $\rightarrow$ B19 phase transition. Picture 1 shows austenite phase prior to transition. On pictures 2-5 we can see nucleation and propagation of martensitic phase. Picture 6 shows stabilized martensite. Molecules are colored according to their local coordination as recognized by Ballviewer: yellow (B2), purple (B19), or blue (B19 with shuffle inversion). The structure recognition algorithm is sensitive to thermal noise, hence austenite appears not to be uniform. A movie of this sequence is available in online supplementary materials. ${ }^{45}$

preferentially nucleates other variants. This shows that the transition is driven by bulk thermodynamics rather than surface reconstruction.

Eventually the B19 regions penetrate the sample and form a series of nanotwinned crystallites with mobile boundaries with B2-like structure. On further cooling these boundaries combine and annihilate as the microstructure coarsens. The remaining boundaries sharpen into twin boundaries.

The crystal structures are close to being compatible such that an austenite-martensite interface can form with singlevariant martensite, however this is not quite realized and we see a further perfectly regular set of twins in each crystallite which accommodate the strain. The B2-B19 transformation requires a shuffle of atoms which is associated with a soft phonon $^{22,37-39}$ at the $\mathrm{M}$ point. Here the shuffle has a longer repeat period corresponding to 15 layers. It is possible to describe this martensite as microtwinned, or having a regular array of stacking faults, or even a modulated crystal structure with a repeated ABABACACACBCBCB stacking. Such a long-period crystal has an energy -0.09660 per atom, compared with -0.09666 for B19, it is not the ground state. We assume that this pattern arises to ensure compatibility with the austenite. In previous work similar stacking faults have been seen, ${ }^{40}$ but in those cases they are irregularly spaced, and their number is determined by constraints placed on the twinning angle by the boundary conditions. Here, with free surfaces, the only constraint comes from the compatibility condition, so the stacking faults are regularly spaced. 
We note that the mismatch is also accommodated by a mesoscopic rotation of the martensite, as seen previously in 2D simulations. ${ }^{30}$

These snapshots show that the results of simulations performed are in good agreement with observations from microscopy and theory. ${ }^{16}$ The most important features of the martensitic phase transition: twinned structure, symmetric variants of martensite, and self-accommodation were clearly recovered. It also shows that nucleation depends on both stress- and temperature-induced transition. We imagine the following mechanism: a first nucleation region is thermally induced, which will cause a local strain and hence generate an elastic wave propagating along the crystal. As the free energy saddle of austenite is shallow, atoms are disturbed by the shockwave and transform into the energetically favorable martensite. There is a small local rotation, which causes further stress and ultimately triggers formation of another variant. This hypothesis seems to be quite reasonable, since the martensitic phase transition is rapid and propagates at velocity similar to speed of sound in a given material. This mechanism generates twins dynamically. Thus twins are being produced by both static and dynamic mechanisms: self-accommodation causes the microtwins separated by the blue stripes, while the strain-induced nucleation gives the larger twins which exhibit most strikingly as the zig-zag on the surface. This hierarchy of macro- and microtwinning has been observed in $\mathrm{NiAl}^{41}$ It is very different to the twin branching predicted by Kohn and Muller. ${ }^{42}$ In their work the only driving force is energy minimization while ensuring compatibility at the interface. This is equivalent to our regular array of stacking fault stripes. We do not see branching in this microstructure: this is consistent with the branching criterion Eq. (64) in Ref. 42]. Our second level of hierarchy arises from twins emanating from different interfaces.

After stabilizing the sample at low temperature, a similar procedure was applied to gradually reheat the system. The system was stable until $T=85$, showing the hysteresis typical of MT. Snapshots taken during phase transition are given in Fig. 2. We notice that the macroscopic shape of the sample is recovered on heating: this is an indication of shape memory, but since no strain has been applied this could be due to a surface facetting effect. Pictures 3 and 4 show that at a local level the very definition of austenite is time dependent. At a given instant (3) the local configuration of each atom is B19 like (12 neighbors). However, the data from the same simulation averaged over 1000 time steps (several phonon oscillation periods) gives B2-like local configurations $(8+6$ neighbors). The retransformation temperature for this potential is close to the melting point, and by $T=92$ the sample has neither local or long-ranged order.

\section{B. Shear of martensite}

In this section we describe the response of the martensite to shear. MOLDY is written following Parrinello-Rahman ${ }^{43}$ with both atomic and supercell degrees of freedom. Although we are not using constant stress boundaries, this structure enables us to impose external strains to the sample. Our 80000 atom sample was stabilized for 10000 time steps at $T=27$,
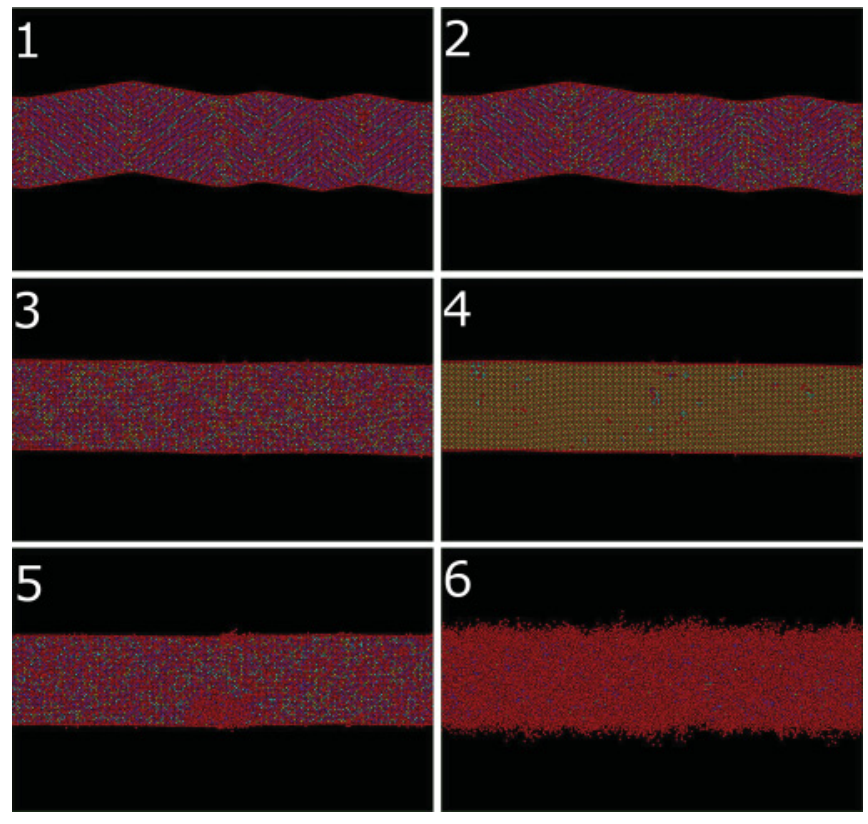

FIG. 2. (Color online) Local crystal structure images for B19-B2 transition. $1 T=84 ; 2 T=86 ; 3$ and 4 correspond to $T=88$, with 3 using a snapshot and 4 using the positions of atoms averaged for 1000 time steps); $5 T=90 ; 6 T=92$.

then sheared by multiplying the matrix of lattice vectors by a volume-conserving deformation matrix:

$$
\mathbf{E}=\left(\begin{array}{ccc}
1 & \epsilon & 0 \\
\epsilon & 1 & 0 \\
0 & 0 & 1-\epsilon^{2}
\end{array}\right)
$$

We used free boundary conditions in the short directions, and periodic boundary conditions in the long direction. The constraint from the periodic, constant shape boundary prevents free rotation and ensures that the sample is sheared without having to resort to fixed atoms. Since the system is under continuous strain we image it using snapshots (Fig. 4).

Snapshot 1 , at $T=64$, shows the sample in B2. The second snapshot shows the same system after being cooled down to $T=27$, all variants are present in equal proportions. Snapshots 3 and 4 show the system being gradually sheared up to $3 \%$. The sample behaves as expected, motion of the twin boundaries to increase the fraction of the variant compatible with the shear.

Once the cold shearing phase is complete, we change from constant shape to constant (zero) stress boundary conditions. This will allow the sample sufficient freedom to recover its initial shape. However, in the martensite phase the system retains its sheared shape, proving that the deformation is indeed plastic.

On heating, images remain similar to 4 up to $T=84$ (snapshot 5) where the austenite appears: notably, the deformation does not change the austenite start temperature. The transition begins at the martensite macrotwin boundaries, and is complete with the shape completely recovered at $T=88$. Melting occurs at $T=90$.

The potential energy for cooling-heating and coolingshearing-heating cycles is shown in Fig. 3. The B2-B19 


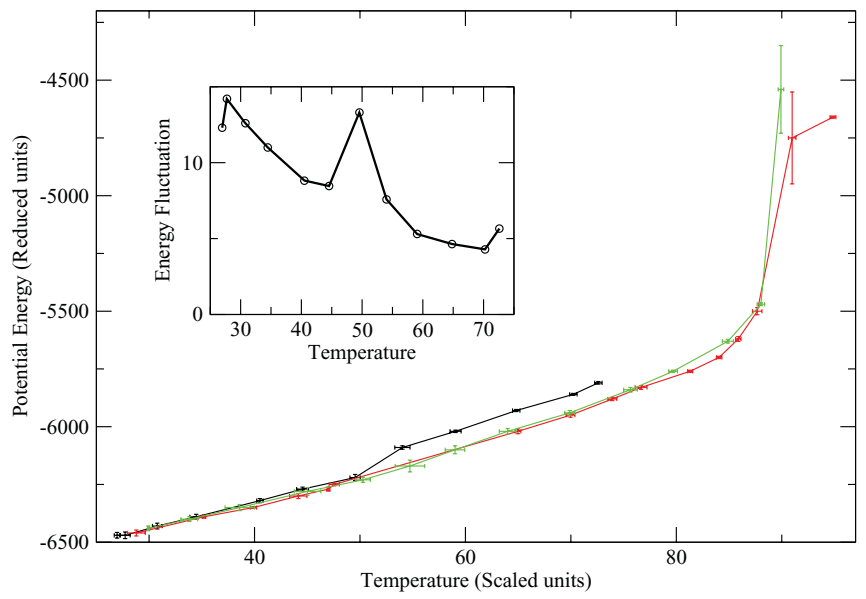

FIG. 3. (Color online) Potential energy vs temperature for an ensemble of 80000 atoms during cooling (black, solid line), heating without deformation (gray, red line), and heating (light gray, green line) after shearing. Errorbars indicate root mean square of fluctuations computed by Moldy. ${ }^{13}$ Inset shows fluctuations in potential energy on cooling, with characteristic peak at the phase transition:

transition occurs at $T=54$ on cooling while the reverse B19-B2 transition starts at $T=84$ and is rapidly followed by melting. Due to thermal noise, it is not possible to follow the transition mechanism directly or define a habit plane.
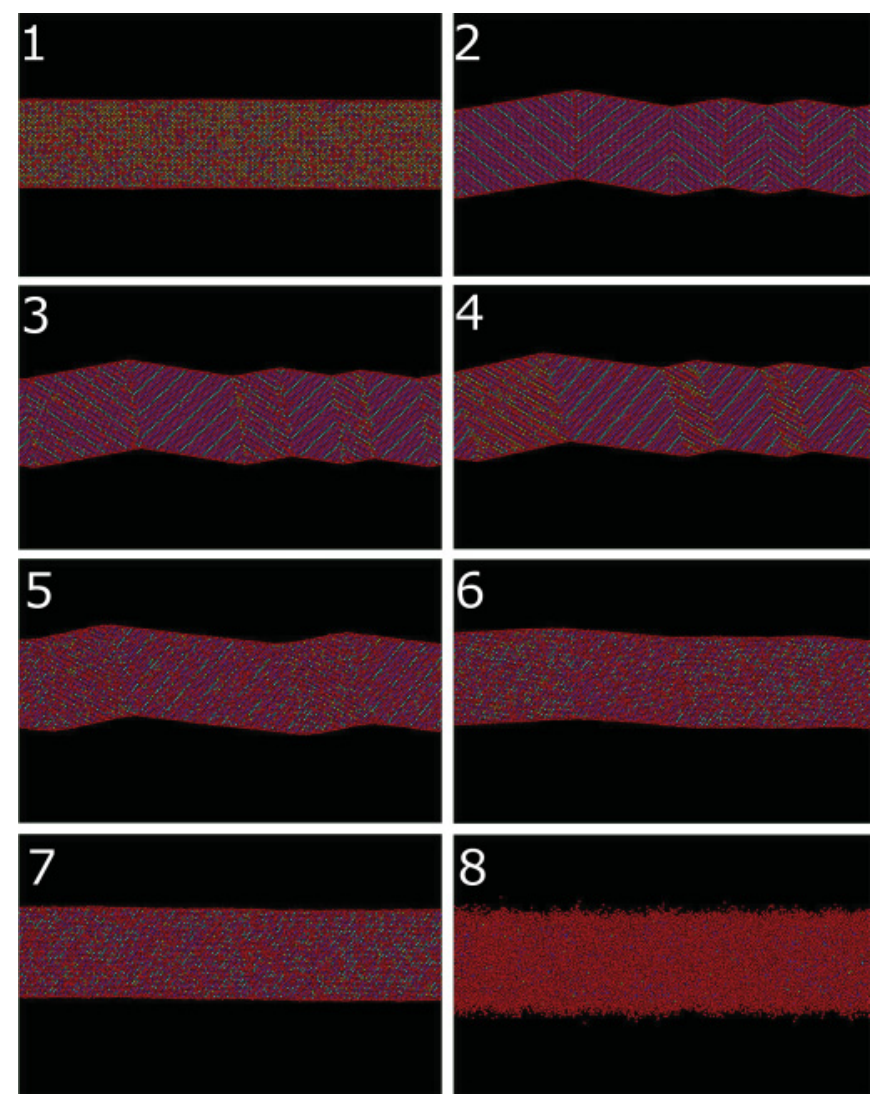

FIG. 4. (Color online) A visualization of the complete cool-loadreheat cycle performed on an ensemble of 80000 atoms. A movie of this sequence is available in online supplementary materials. ${ }^{45}$
The transition can also be seen as a peak in the energy fluctuations corresponding to the divergence of the heat capacity. It is notable that the transition temperature depends on geometry, or more specifically surface energy. For the bar $T=90$ was identified as a melting temperature and above $T=90$ fluctuations in potential energy increase at least by order of magnitude (Fig. 3), which is a consequence of reaching the melting temperature.

The general behavior is reproducible, although there are occasional detectable steps in the energy graph when twin boundaries combine and annihilate.

\section{Stress-induced martensite}

Finally, we examine superelasticity by applying the load above the transition temperature. The sample was first equilibrated at $T=75$ for 10000 time steps (Fig. 5) in the pure austenite phase: no martensite was observed at this stage. To produce each image, the system was equilibrated for 500 time steps and positions were averaged for another 100 time steps in order to reduce thermal noise on the snapshots. A strain tensor with $\epsilon=0.0001$ was applied 800 times at a frequency of once per 600 time steps. The development of the system is shown in Fig. 5. Initially, we have pure
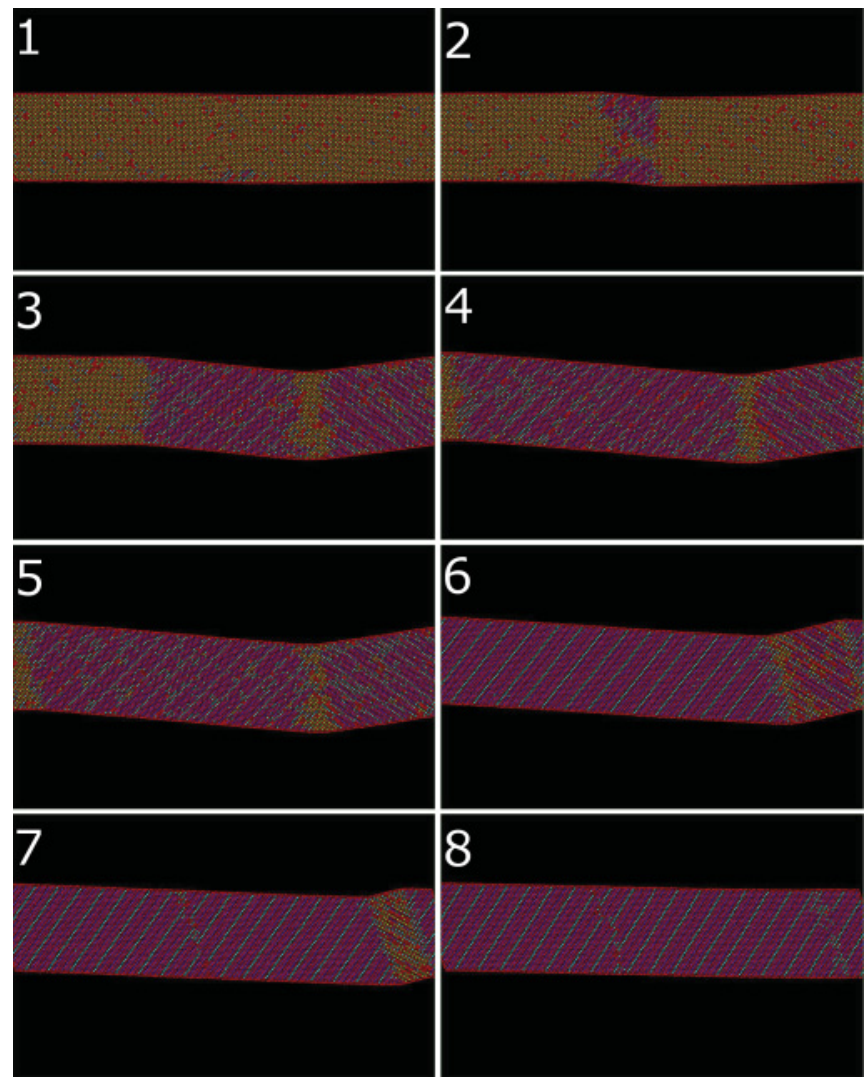

FIG. 5. (Color online) Images of the system being at $T=75$ after applying total shears of $\Sigma \epsilon 0.001,0.003,0.009,0.022,0.035$, $0.051,0.072$, and 0.08 , respectively. Snapshots are noisy at these temperatures, so each image is based on samples where the shearing was stopped for 500 time steps to allow equilibration, and then average positions were taken over 100 time steps. A movie of this sequence is available in online supplementary materials. ${ }^{45}$ 
austenite, and at $0.3 \%$ shear a martensite is nucleated. The coexistence of the martensite and austenite is stable and the volume of martensitic phase is proportional to strain applied. For small strains the pure martensite formation would overcompensate for the external strain, and we find significant stresses within the martensite as shown by the variety of colors. Furthermore, a second variant of martensite is also nucleated: since the applied strain is not the same as the transformation strain this twinned martensite is necessary for full accommodation. By 5\% strain (image 6) the dominant variant of martensite is completely stable. Under further strain (image 7) the boundaries with the minor component merge, by continuous interface movement rather than transformation of entire twins. The periodic boundary conditions plus shear lead to an incompatibility in the nanotwins, which causes another twin boundary to appear. Finally, the transition to a single variant system is shown in snapshot 8 , however the applied and transformation strains are still different, so the martensite cannot be perfect: sure enough two twin boundaries in the crystal structure can be observed.

\section{Conclusions}

We have derived an interatomic potential which, while not describing a specific material, satisfactorily describes the phase transition from B2 to B19 and observed in shape memory alloys such as AuCd. Using this potential we have investigated the martensitic phase transition and its associated microstructure.

From running calculations at many different temperatures we established the thermodynamic transition temperature to be between 70 and 75, depending on boundary conditions. For the freestanding bar we found $T=74$. By contrast, the heating and cooling experiments found considerable hysteresis with the MT at 53-54 on cooling and the reverse transformation at 85-88.

The model also allows us to compare with analytic theories of martensite based on accommodation at the transformation plane $^{3}$ or energy minimization. ${ }^{44}$ We find evidence that microtwinning occurs in accordance with the geometrical theory of martensite. ${ }^{3}$ The observed microtwin structure is not an energy minimizer, since the boundaries are free. Rather, twins are generated dynamically by a build up of torques.

The simulations used relatively small samples to ensure long runs which decoupled the straining/heating time scales from the thermal equilibration ones. The simulated microstructures are in good qualitative agreement with observations from microscopy, exhibiting twinned martensite with a broad interface between austenite and martensite. Martensitic transitions, shape memory, hysteresis in the phase transition, deformation by twin boundary motion, and superelasticity were all demonstrated.

An unexpected and intriguing result was the discovery of a hierarchy of twins. In all samples imaging of local crystal structure $^{34}$ showed a regular array of stacking fault stripes. These are not energetically favored, and we conclude that they are necessary to ensure compatibility between the austenite and martensite. Regarding the regions between stacking faults as nanotwins, these are the twins predicted by the theory of Wechsler, Lieberman, and Read, ${ }^{3}$ but curiously they do not play any role in the shape memory behavior. At a larger scale there are a second level of twins which appear along the bar. These can arise from stress-induced nucleation, and at low temperatures they exhibit atomically sharp boundaries, they move in response to external shear seemingly unimpeded by the nanotwins. Both sets of twins correspond to martensitic variants in the EPN of the austenite. It is this second level of twin which is responsible for the observed shape memory effect.

\section{ACKNOWLEDGMENTS}

The authors thank the Edinburgh Parallel Computing Centre (EPCC) of The University of Edinburgh for the CPU time allocation, EPSRC for support under EP/F010680/1 and Dr. Oliver Kastner for helpful discussions.
${ }^{1}$ L. M. Schetky, Sci. Am. 241, 74 (1979).

${ }^{2}$ D. C. Lagoudas, Shape Memory Alloys-Modeling and Engineering Applications (Springer, New York, 2008).

${ }^{3}$ M. S. Wechsler, D. S. Lieberman, and T. A. Read, AIME Trans. J. Metals 197, 1503 (1953).

${ }^{4} \mathrm{~K}$. Bhattacharya, Microstructure of Martensite (Oxford University Press, Oxford, 2003).

${ }^{5}$ Z. Y. Zhang, R. D. James, and S. Muller, Acta Mater. 57, 4332 (2009).

${ }^{6}$ U. Pinsook and G. J Ackland, Phys. Rev. B 62, 5427 (2000).

${ }^{7}$ T. Uehara, C. Asai, and N. Ohno, Modell. Simul. Mater. Sci. Eng. 17, 3 (2009).

${ }^{8}$ S. Ozgen and O. Adiguzel, J. Phys. Chem. Solids 64, 459 (2003).

${ }^{9}$ S. Ozgen and O. Adiguzel, J. Phys. Chem. Solids 65, 861 (2004).

${ }^{10}$ G. D. Lorenzi and C. P. Flynn, J. Phys. C 18, L769 (1985).

${ }^{11}$ H. S. Park, K. Gall, and J. A. Zimmerman, Phys. Rev. Lett. 95, 255504 (2005).
${ }^{12}$ H. S. Park and C. Ji, Acta Mater. 54, 2645 (2006).

${ }^{13}$ G. J. Ackland, K. D’Mellow, L. S. Daraszewicz, D. J. Hepburn, M. Uhrin, and K. Stratford, Comput. Phys. Commun. 182, 2587 (2011).

${ }^{14}$ Y. Kudoh, M. Tokonami, S. Miyazaki, and K. Otsuka, Acta Metall. 33, 2049 (1985).

${ }^{15}$ S. Rosen and J. A. Goebel, Trans. Metall. Soc. AIME 242, 4, 722 (1968).

${ }^{16}$ M. S. Wechsler, D. S. Lieberman, and T. A. Read, J. Appl. Phys. 26, 473 (1955).

${ }^{17}$ A. Olander, Z. Kristallogr. A 83, 145 (1932).

${ }^{18}$ T. Ohba, Y. Emura, S. Miyazaki, and K. Otsuka, Mater. Trans. Jpn. Inst. Met. 31, 12 (1990)

${ }^{19}$ X. Huang, K. M. Rabe, and G. J. Ackland, Phys. Rev. B 67, 024101 (2003).

${ }^{20}$ X. Huang, G. J. Ackland, and K. M. Rabe, Nat. Mater. 2, 307 (2003).

${ }^{21}$ U. Pinsook and G. J. Ackland, Phys. Rev. B 58, 11252 (1998). 
${ }^{22}$ U. Pinsook and G. J. Ackland, Phys. Rev. B 59, 13642 (1999).

${ }^{23}$ M. I. Mendelev and G. J. Ackland, Philos. Mag. Lett. 87, 349 (2007).

${ }^{24}$ Typically not presented by the authors.

${ }^{25}$ P. M. Morse, Phys. Rev. 34, 57 (1929).

${ }^{26}$ R. S. Elliott, N. Triantafyllidis, and J. A. Shaw, J. Mech. Phys. Solids 54, 161 (2006).

${ }^{27}$ R. S. Elliott, N. Triantafyllidis, and J. A. Shaw, J. Mech. Phys. Solids 54, 193 (2006).

${ }^{28}$ V. S. Guthikonda and R. S. Elliott, Continuum Mech. Thermodyn. 21, 269 (2009); D. S. Karls and R. S. Elliott (private communication).

${ }^{29}$ R. S. Elliott, N. Triantafyllidis, and J. A. Shaw, J. Mech. Phys. Solids 59, 216 (2011).

${ }^{30}$ O. Kastner and G. J. Ackland, J. Mech. Phys. Solids 57, 109 (2009).

${ }^{31}$ O. Kastner, G. Eggeler, W. Weiss, and G. J. Ackland, J. Mech. Phys. Solids 59, 1888 (2011).

${ }^{32}$ D. J. Hepburn and G. J. Ackland, Phys. Rev. B 78, 165115 (2008).

${ }^{33}$ D. J. Hepburn, G. J. Ackland, and P. Olsson, Philos. Mag. 89, 3393 (2009).
${ }^{34}$ G. J. Ackland and A. P. Jones, Phys. Rev. B 73, 054104 (2006).

${ }^{35}$ W. G. Hoover, Phys. Rev. A 31, 1695 (1985).

${ }^{36}$ S. Nose, J. Chem. Phys. 81, 511 (1984).

${ }^{37}$ X. Huang, C. Bungaro, V. Godlevsky, and K. M. Rabe, Phys. Rev. B 65, 014108 (2001).

${ }^{38}$ K. Parlinski and M. Parlinska-Wojtan, Phys. Rev. B 66, 64307 (2002).

${ }^{39}$ K. Parlinski, M. Parlinska, and R. Gotthardt, J. Phys. IV (France) 112, 635 (2003).

${ }^{40}$ G. J. Ackland, A. P. Jones, and R. Noble-Eddy, Mater. Sci. Eng. A 481-482, 11 (2008).

${ }^{41}$ D. Schryvers, P. Boullay, R. V. Kohn, and J. M. Ball, J. Phys. IV 11, 23 (2001).

${ }^{42}$ R. V. Kohn and S. Muller, Philos. Mag. 66, 697 (1992).

${ }^{43}$ M. Parrinello and A. Rahman, Phys. Rev. Lett. 45, 1196 (1980).

${ }^{44}$ J. M. Ball and R. D. James, Arch. Ration. Mech. Anal. 100, 13 (1987).

${ }^{45}$ See Supplemental Material at http://link.aps.org/supplemental/ 10.1103/PhysRevB.84.144113 for movies associated with the figures in the paper. 